\section{The Effectiveness of Transdiagnostic}

Group therapy on Anxiety, Depression and Perfectionism for Mothers of Children with Cerebral palsy
Seyedeh Sara Mousavi, Dr.Abolfazl Mohammadi,
Dr.Mehdi Soleimani

\begin{abstract}
Objective: This study aimed to determine the effectiveness of group therapy protocol transdiagnostic on Anxiety, Depression and Perfectionism were mothers of children with cerebral palsy. Methods: Using purposive sampling, 30 mothers of children with cerebral palsy who meet the criteria for entering the sample were selected and randomly divided into two groups of 15 experimental and control. Quasiexperimental study with pretest-posttest control group. To collect data and Anxiety Depression Scale (DASS-42; Lvvyband and Lvvyband, 1995) and Ahvaz Perfectionism (APS; Najarian, protecting and Smith, 1378) was used. Experimental group received eight 90-minute sessions once a week, under therapy were transdiagnostic. Results: The results of covariance analysis and independent t-test showed that the therapy resulted in a reduction of symptoms of anxiety, depression and perfectionism are mothers of children with cerebral palsy. Conclusions: The effectiveness of group therapy transdiagnostic on anxiety, depression and perfectionism mothers of children with cerebral palsy was approved. Given the importance of the mother's mental health and its impact on children can help create the conditions can be achieved.
\end{abstract}

Keywords: transdiagnostic, anxiety, depression, perfectionism, cerebral palsy

\section{Introduction}

Health officials have always been a subject of considerable attention to chronic diseases in all age groups, socio-economic and cultural, respectively. Cerebral palsy is the most common chronic disability and neurological complications in children (Prvdnt, Barbosa, Ray, 2010). Some researchers, the number of people with cerebral palsy to over 167 million by 2050 and 797 billion in health care costs they estimate (Mir Safavi, fesharaki, Farhad, 2008). Children with Cerebral Palsy major limitations in activities of daily life. These limitations have led to the need for long-term care that children need to grow beyond its natural growth. Child care is the responsibility of parents, but this role in children with functional limitations and longterm dependence is very different (Altmj, Icahn, Akan, Kvksal, Erkin, Age, 2007). Thus, the child's disability affects not only a child's life (Mubarak Khan, Manyr time, Kvnachy, 2000). But that requires special care and support of a member of the family may be different, especially "Mother" focused on returns. (Prvdnt et al., 2010). Studies also have shown that physical health and psychological care for children with cerebral palsy can affect caregivers often are mothers (Davis, Shelley, Waters, Boyd, Cook, Davrn, 2010, Rayna, Donnelly, Rosenbaum, Birhan, Walters, 2005). Parents of disabled children compared with parents of normal children less mental health, anxiety, emotional stress and lower selfesteem and show more stress. (Vaysbrn, 1980; Trott, 1983; Kazak and Marvin, 1984; Bristol et al., 1988; Goldberg et al., 1990; Beckman, 1991; Miller et al., 1992; Plchat et al., 1999; Hsynnzhad, 1375; sajedi and others , 1389; Naraqi and rare Saif, 1381; Peterson and Albers, 2001; Rose, David Jones, 2003). Research also shows that parents of children with disabilities are vulnerable to stress and pressure. Depression is much more than 70 percent of mothers and $40 \%$ of fathers of children with severe disabilities has been observed (Gabta and Singhal, 2005). Other studies have shown that parents of such children are more vulnerable to dangers and 
difficulties that this causes separation or divorce of communication between them (Trott, 1983; Dalayr, 1984; urgent and Las Wan, 1984). Although it is recognized that some families might very well with children with disabilities compatible, but the presence of a disabled child devastating effect on most parents must therefore interventions effective and has greater flexibility to be used to according to their mental tional step to try to improve the mental health of the harvest. Is to reduce these problems; Btshav (1997) believes that using the methods of cognitive problems, experts can help families with disabled children, help with stress, depression, conflict and loneliness deal, especially when the feel capable to help parents and childcare weakening. In recent years, effective treatments for emotional disorders, especially anxiety and depression has been introduced (Butler, Chapman, Forman \& Beck, 2006), but a significant number of people do not have access to them. Norton and Hop (2005) believe that "the issue is not related to existing treatments, but from the manner of their delivery." So far, guidelines and protocols on the cognitivebehavioral therapy for emotional disorders have published many studies and many have confirmed the effectiveness of this turn (Butler et al., 2006). However, he mentioned the slight expansion between therapists and availability they lack for clients, the problem is in the way (and Philip Norton, 2008). Transdiagnostic cognitive behavioral therapy (turned integrated; Barlow et al., 2011) In response to the limitations listed.

\section{Methods:}

Samples of this study were selected based on purposive sampling and available sample. In this study, after advertising in some rehabilitation centers in Tehran and introduced into schools in Tehran, A number of 30 persons who fulfill the entry criteria were enrolled and randomly assigned 15 patients were divided evenly into two groups. At first the DASS-42 scale for anxiety and depression scale by Lvvyband and Lvvyband (1995) was produced with Article 42 for each subscale is determined to Article 14 and 21 of the short form is also available. The validity of research results and a good reputation for this tool has reported Cronbach's alpha for subscale of depression, anxiety and stress, respectively, 91/0, 84/0 and 90/0 and correlation with the Beck Depression Inventory and Beck Anxiety Inventory 81 tool / 0 and 74/0 reported. The Persian version of this tool by the owners and others (1384) was validated in Iran and indexes by using factor analysis and criterion validity, and reliability by internal consistency (77/0 depression, anxiety 78/0) in Iranian population is valid and scale (APS) Ahvaz perfectionism by Najarian, protecting and Goldsmith (1378) to measure perfectionism made. The scale based on analysis of the responses of male and female subjects martyr Chamran University of Ahvaz Islamic Azad University was built. This is a self-report scale that 27 material. Answer upon the whole continuum often, sometimes, rarely or never ranked in order of scores of zero, 1, 2, 3 to each option has been allocated. To assess the internal consistency of the scale Cronbach's alpha coefficient was used. Based on the results of Cronbach's alpha coefficients for all subjects vary from 850 subjects daughter, $89 / 0$, and $81 / 0$ is a boy. The correlation coefficients between the scores of the subjects in the test and retest $68 / 0,69 / 0$ female subjects, and subjects son is $67 / 0$ to be acceptable psychometric properties have been reported (Najarian et al., 1378). To validate this scale is a scale model of the disease Type A, Type A nonconsensual and somatization subscale scale revised list of psychiatric symptoms (SCL90-R) research model was presented to students. Reliability coefficients were 
calculated with a scale model of type A disease-scale 65/0 and 41/0 physical complaints that were significant both at 0.05. Researchers Based on the results obtained, Ahvaz Perfectionism Scale convenient and credible tool for detecting features of perfectionism report (Najarian et al., 1378). Implemented and those under Depression Scale score of 10 or higher on the anxiety subscale score of 8 or higher were followed by an explanation about the investigation and its terms and other ethical and informed consent, they were included after completing treatment and again after 3 months on the attendees were both scale. The control group, see also such as the experimental group in initial assessments meetings or pre-test and post-test or final evaluation. They were waiting for treatment transdiagnostic. Then the experimental group received 8 weekly sessions of group training transdiagnostic according to the program were proposed. In this study, 2 sessions absence of meetings was allowed, the absence of 3 sessions was determined as the criterion loss. After completion of therapy sessions were running again research tools (DASS and APS) and after 3 months, the participants were invited to complete this tool.

Results

T-test for independent groups to examine age differences between experimental and control groups were used. The results of that there is no significant difference between the two groups in terms of age. $(146 / 0=\mathrm{P}$; $267 / 0=\mathrm{t} ; 333 / 0=$ Mdif $)$. Compare groups using Chi-square test showed no significant difference between the two groups (59 / OP $=; 04 / 1 \lambda 2=)$. Based on independent T-test, the average pre-test in each of the variables studied two groups was not significant (Table 1). Therefore, the random assignment process before the intervention is confirmed. Shapiro-Wilk normality was used to check the score of the test. Hare et al (1998) suggest that when the scores from this level $(5 / 2 \pm=\mathrm{Z})$ exceed the given remote (Perth) considered and should be deleted according to information provided by one of the participants in the group that's why the trial was removed from the population and was referred to drug treatment. Distribution of scores in three stages in anxiety, depression and perfectionism normal curve is not significantly different $(\mathrm{P}>0.05)$. Before the analysis of covariance assumption of homogeneity of variances was tested by Levene test. In cases where the original assumptions such as homogeneity of variance and covariance analysis or regression coefficient $t$ test was not confirmed to the difference between the means used to analyze the results. Transdiagnostic analysis of covariance showed that the treatment group after group meetings and three-month follow-up was no significant difference in depression. With regard to pre-test scores as covariate results and three months follow-up after completion of treatment for depression is statistically Mnadarbvdh (Table 2). The results of analysis of covariance showed that after the meetings the group had no significant difference in anxiety scores (Table 3 ). According to the results of the 3-month follow-up has not homogeneity of variance, independent t-test was used and no significant difference in scores was seen after three months (Table 4).

Table (1) compares the average pre-test experimental and control groups using independent $t$ test 
Discussion

This study aims at the overall effectiveness of group therapy transdiagnostic in reducing anxiety, depression and perfectionism were the mothers of children with cerebral palsy. The results show the effectiveness of the treatment group was transdiagnostic. Transdiagnostic group therapy resulted in significant differences in pre-test and posttest on all measures studied groups in the study. Then transdiagnostic hypotheses about the effectiveness of group therapy in reducing anxiety, depression and perfectionism were analyzed. Overall results are consistent with the claims of the treatment and research of (ard et al., 2010), Bvys.h et al. (2012) and Farkyvnh et al (2012) based on the performance of unified transdiagnostic treatment in patients with disorders The results also show that the protocol Pkparchh managed in accordance with the logic good situation for creating it and in line with the claim creators Based on the target of transdiagnostic joint (Vylamvska et al., 2010) significant change in the operating perfectionism clinically and statistically creation he does. The results of this study show that clinicians may be due to the treatment rather than the use of guides numerous health can approach the sparing more design and use of such measures, which require the use of manual therapy Akhtsasy_ Dysfunctions and treatment to relieve heavy (Mansell, Harvey, Watkins and Schaefer, 2009).

The success of this approach in the treatment of patients with emotional disorders in this study and previous research and future can be argued valuable for the dissemination of evidence-based treatment and obstacles in the implementation of them, such as the time and cost required Help solve various therapeutic for proper training. In addition, clinicians need when dealing with patients with complex clinical protests instead of using multiple protocols with a protocol eliminates the unit.

This study has been limited. Although significant findings of smaller samples is more accurate, but because of the limited number of samples in group therapy decreases the external validity of research. In future intervention studies with larger samples can compensate for these shortcomings. Surveys conducted only on mothers and also the limitations of this research. Since there was no difference in the stress and turmoil of parents is suggested that this intervention on the children's fathers also take place. It is suggested that future studies transdiagnostic interventions in the comprehensive prevention measures and the choice to use. General education and intervention for families of children with cerebral palsy in children, and to accept the first diagnosis it is very necessary. The study of this treatment in different subjects such as children and the elderly and other chronic diseases and at different levels, such as school, family or multilevel done. Flanders represent but the Vlnsky (2004) believe that it is necessary for the performance of preventive studies done. They are of the opinion that research on the protective factors, mediating emotional disorders to be done. In addition, long-term follow-up results of future studies suggest that group therapy transdiagnostic. Transdiagnostic and efficacy studies of treatment for problems and other disorders of the circuit is required (ard et al., 2010). Finally, it seems transdiagnostic more research to compare interventions with specific treatments and other disorders need.

\section{References}

Hosseini Nejad, M. (1375). Study of Stress and parental marital educable mentally retarded children with parents of normal children and children with disabilities 
sensorimotor Tehran, Master's thesis.

Faculty of Education and Psychology, Allameh Tabatabaei University.

Sajedi, F., Vameghi, g., Alizadeh, and., King Khosravi, Gh., Karimloo, M., Ravryan, a., Shahshahani Poor's, S., (1389). Is anxiety in mothers of children with cerebral palsy is higher than normal children ?, Journal of Rehabilitation, 1389 No. 5, pp. 20-15.

Saif Naraqi, m., Rare, S., (1381). Stress and parents of children with disabilities, special education journal, 10 and 11, pp. 23-19.

Mohammadi, A.; Birashk, b. And Gharraee, b., (1392). Transdiagnostic compare the effectiveness of group therapy and group therapy, cognitive, emotional regulation processes. Iranian Journal of Psychiatry and Clinical Psychology, Volume 19 _ Number 3, Pages 194-187.

Mohammadi, A.; Birashk, b. And Gharraee, b., (1392). Comparative effectiveness of group therapy in reducing symptoms of anxiety and depression transdiagnostic: 3-month follow-up. Psychological research, _ Volume 16, Issue 1, pages 23-7.

Altmdag, Ö, Içcan, A., Akcan, S., Köksal, S., Erçin, M., Ege, L. Anxiety and depression levels in mothers of children with cerebral palsy. Turk Fiz Tip Rehab Derg; 4, 22-53.

Barlow, D.H., Ellard, K.K., Fairholme, C.P., Farchione, T.J., Boisseau, C.L. Allen, L.B. \& Ehrenreich-May, J. (2011).The unified protocol for transdiagnostic treatment of emotional disorders: Client workbook. New York, NY: Oxford University Press.
Beckman, P. J. (1991). Comparison of Mother's and Father's Perception of the Effect of Young Children with and Without Disabilities. American Journal on Mental Retardation. Vol. 95, No. 5, 585-595.

Bristol, M. Gallagher, J. J. et Schopler, E. (1988). "Mthers and fathersof young developmentally disabled and nondisabled boys: Adaptation and spousal support". Developmental psychology, 24, p. 441-451.

Butler, A. C., Chapman, J. F., Forman, E. M., \& Beck, A. T. (2006). The empirical status of cognitive-behavioral therapy: A review of meta-analyses. Clinical Psychology Review, 26, 17-31.

Davis E, Shelly A, Waters E, Boyd R, Cook K, Davern M. (2010). The impact of caring for a child with cerebral palsy: quality of life for mothers and fathers. Child: Care, Health and Development; 36(1):63-73.

Egan, S. J., Wade, T. D., Shafran, R. (2011). Perfectionism as a transdiagnostic process: A clinical review, 2, 203-212.

Goldberg, L. R. (1991). Is there a basic level of personality description? Journal of Personality and Social Psychology, 60, 348361.

Guptu, A. Singhal, N. (2005). Psychosocial Support for Families of Children with Autism. Asia Pacific Disability

Rehabilitation Journal, Vol. 16.2, 62-83.

Kazak AE, Wilcox BL. (1984). The structure and function of social support networks in families with handicapped 
children. American Journal of Community

Psychology 12(6): 645-61.

Mir Safavi M., Fesharaki M., Farhadi S.

(2008). Determine of association quality of

life and stress coping method among the

caregiver of patient with multiple sclerosis.

Medical Sciences Journal, 10(5): 563-72.

Mobarak R., Khan N., Munir S., Zaman

S., McConachie H. (2000). Predictors of

stress in mothers of children with cerebral

palsy in Bangladesh. Journal of Pediatric

Psychology; 25(6):427.

Norton, P. J., \& Hope, D. A. (2005).

Preliminary evaluation of a broad-spectrum cognitive- behavioral group therapy for anxiety. Journal of Behavior Therapy and Experimental Psychiatry, 36, 79-97.

Prudente, COM., Barbosa, MA. Porto, CC. (2010). Relation between quality of life of mothers of children with cerebral palsy and the children's motor functioning, after ten months of rehabilitation. Revista LatinoAmericana de Enfermagem; 18(2):149-55.

Raina, P., O'Donnell, M., Schwellnus, H., Rosenbaum, P., King, G., Brehaut, J. (2004). Caregiving process and caregiver burden: conceptual models to guide research and practice. BMC Pediatrics 4(1): 1. 
